\title{
Mitral Annular Dynamics and Left Ventricular Diastole
}

\author{
Angel Lopez-Candales ${ }^{\mathrm{a}, \mathrm{d}}$, Dagmar F. Hernandez-Suarez ${ }^{\mathrm{b}}$, \\ Francisco Lopez Menendez ${ }^{\mathrm{C}}$
}

\begin{abstract}
Background: Though diastolic interrogation of the mitral annulus (MA) using tissue Doppler imaging (TDI) has been quite useful in assessing left ventricular (LV) diastolic function, the relative contribution of the previous cycle MA systolic displacement or velocity components to subsequent LV diastole has not been previously investigated. We therefore sought to determine the association between MA systolic dynamics and LV diastolic function parameters.
\end{abstract}

Methods: For this retrospective study, only complete echocardiograms having good endocardial border resolution of both left atrial (LA) and LV chambers with M-mode (MAPSE) and tissue Doppler of the lateral MA (MA TDI S') as well as complete Doppler data to perform assessment of LV diastole were included in our analysis.

Results: Data from 100 patients (mean age $54 \pm 14$ ) showed that both MA systolic displacement and velocity correlate with LV ejection fraction $(\mathrm{P}<0.05)$. Only MA displacement was associated with age and LV mass. Most importantly no correlation was found between MAPSE and LV diastole. However, in sharp contrast MA TDI S' correlated with MA relaxation velocities during both early and late LV diastole.

Conclusion: Even though MAPSE and MA TDI S' are surrogate measures of LV ejection fraction, only MA TDI S' correlates with diastolic MA velocities as no correlation was identified between MAPSE and measures of LV diastole. Additional studies are now warranted to explore whether a reduced MA TDI S' may affect the symptomatic profile or the overall prognosis of patients with LV diastolic dysfunction.

Keywords: Echocardiography; Mitral annulus; Diastolic dysfunction

Manuscript submitted August 30, 2017, accepted September 25, 2017

${ }^{a}$ Cardiovascular Medicine Division, University of Puerto Rico School of Medicine, San Juan, Puerto Rico

${ }^{b}$ Department of Medicine, University of Puerto Rico School of Medicine, San Juan, Puerto Rico

'Division of Cardiovascular Health and Disease, University of Cincinnati College of Medicine, Cincinnati, OH, USA

${ }^{\mathrm{d}}$ Corresponding Author: Angel Lopez-Candales, Cardiovascular Medicine Division, University of Puerto Rico School of Medicine, Medical Sciences Building, PO Box 365067, San Juan 00936-5067, Puerto Rico.

Email: angel.lopez17@upr.edu

doi: https://doi.org/10.14740/cr602w

\section{Introduction}

Three continuous layers of muscle bundles arranged in a helical configuration are known to comprise the ellipsoid shape of the normal left ventricle (LV) [1-4]. Even though all muscle bundles are required for optimal mechanical efficiency [5], longitudinal fiber shortening in systole causes basal to apical displacement of the mitral annulus (MA), while rapid lengthening in diastole is responsible for reversing the direction of MA displacement to its resting basal location [5].

From a hydraulic perspective, based on the Pascal's principle, MA displacement not only should assist in LV systolic function, but also MA apical to basal motion should aid in LV filling [6]. While maximal MA plane systolic excursion (MAPSE) and MA systolic velocity have been used as potential measures of LV systolic function $[7,8]$ in certain clinical scenarios, both measures have limited diagnostic utility [4]. Though interrogation of the MA diastolic to determine early and late diastolic relaxation velocities with the use of tissue Doppler imaging (TDI) has been quite useful in assessing LV diastolic function [9], the relative contribution of the previous cycle MA systolic displacement or velocity component in regards to subsequent LV diastole cycle has not been previously investigated. We therefore sought to determine the association between MA dynamics and diastolic function parameters.

\section{Methods}

We therefore conducted a retrospective analysis and queried our University Hospital Medical Center, Cincinnati, OH echocardiography database for complete studies having good endocardial border resolution of left atrial (LA) and LV chambers from the parasternal long axis, two- and four-chamber apical views, M-mode and tissue Doppler (TDI) of the lateral MA as well as complete collection of required Doppler variables to perform assessment of LV diastolic function so that we could be able to determine which MA component, motion or velocity correlates best with LV diastolic function. Studies were excluded if ectopy or arrhythmias were present as well as if the heart rate was greater than 100 beats per minute. The University of Cincinnati Institutional Review Board (IRB \# 12061302) approved the study.

All two-dimensional echocardiographic studies were performed using commercially available systems (Vivid 7 and 9 , GE Medical Systems, Milwaukee, WI, USA). Images were 
Table 1. Echocardiographic Data of the Study Population

\begin{tabular}{lll}
\hline Variables & Mean & Range \\
\hline Age $($ years $)$ & $53 \pm 14$ & $24-89$ \\
\hline BSA $\left(\mathrm{m}^{2}\right)$ & $2.0 \pm 0.3$ & $1.4-3.2$ \\
LVMI $\left(\mathrm{g} / \mathrm{m}^{2}\right)$ & $108 \pm 41$ & $45-234$ \\
LAVI $\left(\mathrm{mL} / \mathrm{m}^{2}\right)$ & $27 \pm 11$ & $10-76$ \\
MAPSE $\left(\mathrm{cm}^{2}\right)$ & $1.2 \pm 0.3$ & $0.3-1.9$ \\
LVESV $\left(\mathrm{cm}^{3}\right)$ & $51 \pm 46$ & $5-246$ \\
LVEDV $\left(\mathrm{cm}^{3}\right)$ & $125 \pm 51$ & $38-329$ \\
LVEF $(\%)$ & $64 \pm 18$ & $11-93$ \\
MV E $\left(\mathrm{cm} / \mathrm{s}^{3}\right)$ & $82 \pm 25$ & $34-183$ \\
MV A $(\mathrm{cm} / \mathrm{s})$ & $74 \pm 32$ & $10-168$ \\
MV E/A ratio & $1.4 \pm 1.2$ & $0.4-10.6$ \\
MA TDI S' $(\mathrm{cm} / \mathrm{s})$ & $7.8 \pm 2.6$ & $3-14$ \\
MA TDI E' $(\mathrm{cm} / \mathrm{s})$ & $8.6 \pm 3.8$ & $2-21$ \\
MA TDI A' $(\mathrm{cm} / \mathrm{s})$ & $8.3 \pm 3.2$ & $2-21$ \\
MV E/MA TDI E' ratio & $11.8 \pm 7.8$ & $3.6-53.0$ \\
\hline
\end{tabular}

obtained in left lateral decubitus position with the patient in the supine position using a $3.5-\mathrm{MHz}$ transducer. Standard two-dimensional, color, pulsed, and continuous-wave Doppler data were digitally acquired in gently held end-expiration, and saved in regular cine loop format for subsequent offline analysis (EchoPAC version 111.0.00; GE-Vingmed Ultrasound AS).

For the particular purpose of this study, LV end-systolic and end-diastolic volumes were measured as recommended by the American Society of Echocardiography to determine LV ejection fraction using the Simpson's method [4]. In addition, surrogate measures of LV systolic function such as MAPSE as well as its systolic ( $\left.\mathrm{S}^{\prime}\right)$ velocity of the lateral portion of the MA were acquired as previously described [4]. LA volumes were calculated using the biplane area-length formula, while LV mass was determined using M-mode echocardiography, as previously described [4]. Both LA volumes and LV mass were corrected for body surface area [4]. Finally, LV diastolic function was determined using recommended American Society of Echocardiography guidelines [9].

All continuous data are listed as mean \pm standard deviation (SD). Correlations between MAPSE as well as MA TDI S' and other collected echocardiographic parameters were performed using Pearson's correlation. Multivariate logistic regression analysis was performed to detect the best parameter that identified MAPSE and MA TDI systolic velocity. P-values of $<0.05$ were considered to be statistically significant. All analyses were performed using SAS version 9.4 (SAS Institute).

\section{Results}

The studied population included data from 100 patients (mean
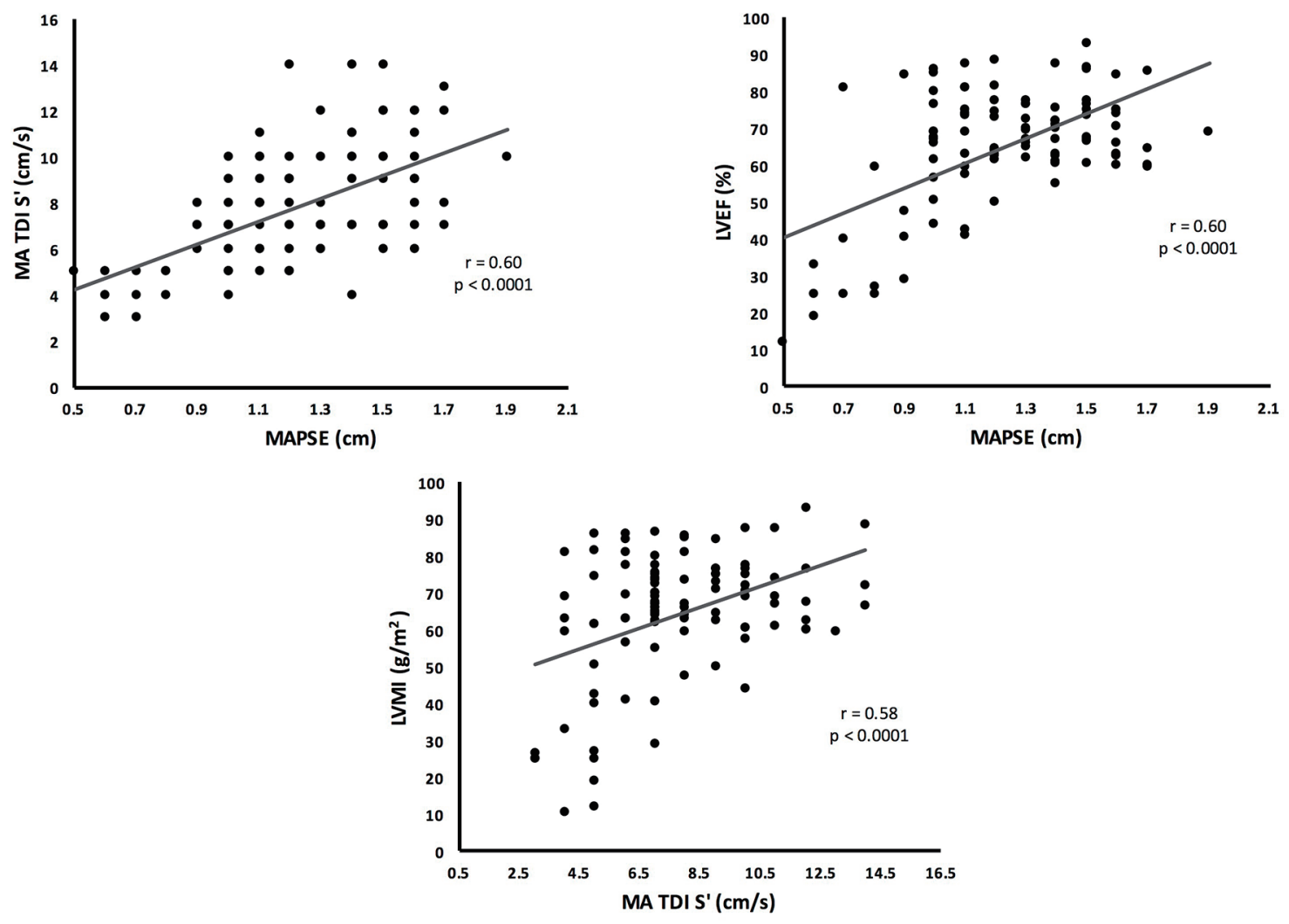

Figure 1. Pearson correlation analysis showing the echocardiographic variables better associated with MAPSE and MA TDI S'. 
Table 2. Multiple Linear Regression Analysis Showing the Association Between MAPSE and MA TDI S' With the Echocardiographic Parameters

\begin{tabular}{|c|c|c|c|c|}
\hline \multirow{2}{*}{ Variables } & \multicolumn{2}{|c|}{ MAPSE } & \multicolumn{2}{|c|}{ MA TDI S' } \\
\hline & Coefficient & P-value & Coefficient & P-value \\
\hline Age (years) & -0.006 & 0.003 & -0.005 & 0.770 \\
\hline BSA & -0.005 & 0.953 & -0.465 & 0.457 \\
\hline LVMI & -0.002 & 0.017 & -0.005 & 0.340 \\
\hline LAVI & 0.004 & 0.097 & -0.020 & 0.269 \\
\hline MAPSE & - & - & 1.837 & 0.028 \\
\hline LVESV & -0.008 & $<0.001$ & 0.030 & 0.070 \\
\hline LVEDV & 0.005 & $<0.001$ & -0.022 & 0.019 \\
\hline LVEF (\%) & -0.003 & 0.419 & 0.025 & 0.298 \\
\hline MV E & -0.001 & 0.562 & -0.018 & 0.068 \\
\hline MV A & 0.001 & 0.582 & -0.004 & 0.667 \\
\hline MV E/A ratio & -0.009 & 0.793 & -0.185 & 0.480 \\
\hline MA TDI S’ & 0.031 & 0.028 & - & - \\
\hline MA TDI E' & 0.004 & 0.762 & 0.425 & $<0.001$ \\
\hline MA TDI A' & 0.011 & 0.198 & 0.243 & $<0.001$ \\
\hline MV E/MA TDI E' ratio & 0.002 & 0.783 & 0.008 & 0.181 \\
\hline
\end{tabular}

age $54 \pm 14,53$ of these patients were males with a mean body surface area of $2.0 \pm 0.3$ ). Mean, SD and range of measurements for all the intended echocardiographic variables used for this study are shown in Table 1. Significant association was found between LVMI, LAVI, LVEF, MV E/A ratio, MA TDI E', MA TDI A', MV E/MA TDI E' ratio with both MAPSE and MA TDI S'. Additionally, age was associated with MAPSE and MVE with MA TDI S' $(\mathrm{P}<0.05)$. Figure 1 depicts the echocardiographic variables showing the strongest association with MAPSE and MA TDI S'. Multiple linear regression analysis showing the association between MAPSE and MA TDI S' with the studied echocardiographic parameters is shown in Table 2.

\section{Discussion}

Cardiac performance is directly related to its architecture. In the case of the LV, longitudinal and circumferential myocardial fiber orientation facilitates systolic ejection during fiber shortening and filling in diastole with rapid fiber lengthening [1-5]. Furthermore, LV longitudinal fibers also contribute to LV systole and diastole by facilitating MA displacement [5]. Based on these anatomical and functional principles, the MA should experience dynamic changes throughout the cardiac cycle; therefore, each preceding LV diastole cycle should impact the ensuing motion or velocity of the MA during systole.

When examining our results, as expected both MAPSE and MA TDI S' correlate with systolic parameters of LV function. In the case of MAPSE, there is a clear dependence on age and LVMI. However, the most notable findings of this study relate to the behavior of the MA with regard to parameters of LV diastolic function. Specifically, no correlation was seen in terms of MAPSE. In sharp contrast, MA TDI S' correlated with MA relaxation velocities during both early and late LV diastoles.

From a mechanistic point of view, LV myocardial fiber architecture creates complex patterns of non-homogeneous of contractility that cannot be depicted by M-mode interrogation of the MA despite the high sampling rate of this imaging modality. Hence, the association between MAPSE and LV ejection fraction is valid only in patients with either normal or dilated LV $[10,11]$ as MAPSE is known to lose its diagnostic value when assessing LV ejection fraction in patients with LV hypertrophy as longitudinal shortening is greatly compromised in these patients [12]. In sharp contrast, TDI has diagnostic advantage in this particular scenario, as it is able to assess specific components of MA displacement such as velocity, acceleration/deceleration and time intervals $[4,9]$. Direct comparisons of these two imaging modalities to assess overall MA displacement revealed that TDI was better than M-mode as it resulted in greater concordance and less observer variability [13]. Since LV twist represents the mean longitudinal gradient of the net difference between clockwise and counterclockwise rotations of the LV apex and base, as viewed from LV apex $[14,15]$, both MAPSE and MA TDI S' values should reflect LV systolic performance [4]. Nonetheless, when considering LV diastole MA TDI S' will be less hampered than MAPSE as TDI rather than M-mode has been a well validated modality to measure relaxation velocities that have become quite useful in assessing LV diastolic function [9]. Finally, TDI allows measurement of all cardiac events (preejection, systole, isovolumic relaxation and diastole) on one acquired image.

Several limitations need to be acknowledged. First, it has retrospective nature of a small sample size. Second, our results 
may not be generalizable to patients with arrhythmias and/ or significant valvular disease, as they were excluded. Third, there is lack of speckle tracking imaging information that might have impacted MA assessments of LV diastolic function. Finally, although recommendations for the evaluation of LV diastolic function by echocardiography have been recently updated [16], yet most of LV diastolic dysfunction patients remained under the same classification by new guidelines. Regardless, both echocardiographic measurements of MAPSE and MA TDI S' remain unchanged; therefore, we believe this has no significant bearing on our results.

\section{Conclusion}

Our study highlights apparent differences in the behavior of MA longitudinal displacement depending on the imaging tool used for interrogation showing the tight known interdependence exiting between LV systole and diastole. Additional studies are now warranted to explore whether a reduced MA TDI $\mathrm{S}$ ' may affect the symptomatic profile or the overall prognosis of patients with LV diastolic dysfunction.

\section{Acknowledgments}

This publication was partially supported by the National Institute of Health Award Numbers R25MD007607, S21MD001830 and TL1 TR001434. Its contents are solely the responsibility of the authors and do not necessarily represent the official views of the National Institutes of Health.

\section{Conflict of Interest}

None.

\section{References}

1. MacIver DH. A mathematical model of left ventricular contraction and its application in heart disease. In: Atherton M, Collins M, Dayer M, editors. Repair and redesign of physiological systems. Boston: WIT Press; 2008. pp. 65-86.

2. MacIver DH. Current controversies in heart failure with a preserved ejection fraction. Future Cardiol. 2010;6(1):97111.

3. Anderson RH, Ho SY, Redmann K, Sanchez-Quintana D, Lunkenheimer PP. The anatomical arrangement of the myocardial cells making up the ventricular mass. Eur J Cardiothorac Surg. 2005;28(4):517-525.

4. Lang RM, Badano LP, Mor-Avi V, Afilalo J, Armstrong A, Ernande L, Flachskampf FA, et al. Recommendations for cardiac chamber quantification by echocardiography in adults: an update from the American Society of Echocardiography and the European Association of Cardiovascular Imaging. J Am Soc Echocardiogr. 2015;28(1):1-
39 e14.

5. Maciver DH. The relative impact of circumferential and longitudinal shortening on left ventricular ejection fraction and stroke volume. Exp Clin Cardiol. 2012;17(1):511.

6. Maksuti E, Carlsson M, Arheden H, Kovacs SJ, Broome M, Ugander M. Hydraulic forces contribute to left ventricular diastolic filling. Sci Rep. 2017;7:43505.

7. Matos J, Kronzon I, Panagopoulos G, Perk G. Mitral annular plane systolic excursion as a surrogate for left ventricular ejection fraction. J Am Soc Echocardiogr. 2012;25(9):969-974.

8. Park YS, Park JH, Ahn KT, Jang WI, Park HS, Kim JH, et al. Usefulness of mitral annular systolic velocity in the detection of left ventricular systolic dysfunction: comparison with three dimensional echocardiography data. J Cardiovasc Ultrasound. 2010;18(1):1-5.

9. Nagueh SF, Appleton CP, Gillebert TC, Marino PN, Oh JK, Smiseth OA, Waggoner AD, et al. Recommendations for the evaluation of left ventricular diastolic function by echocardiography. J Am Soc Echocardiogr. 2009;22(2):107-133.

10. Onose Y, Oki T, Mishiro Y, Yamada H, Abe M, Manabe $\mathrm{K}$, Kageji $\mathrm{Y}$, et al. Influence of aging on systolic left ventricular wall motion velocities along the long and short axes in clinically normal patients determined by pulsed tissue doppler imaging. J Am Soc Echocardiogr. 1999;12(11):921-926.

11. Fang ZY, Leano R, Marwick TH. Relationship between longitudinal and radial contractility in subclinical diabetic heart disease. Clin Sci (Lond). 2004;106(1):53-60.

12. Koulouris SN, Kostopoulos KG, Triantafyllou KA, Karabinos I, Bouki TP, Karvounis HI, Omran H, et al. Impaired systolic dysfunction of left ventricular longitudinal fibers: a sign of early hypertensive cardiomyopathy. Clin Cardiol. 2005;28(6):282-286.

13. de Knegt MC, Biering-Sorensen T, Sogaard P, Sivertsen J, Jensen JS, Mogelvang R. Concordance and reproducibility between M-mode, tissue Doppler imaging, and two-dimensional strain imaging in the assessment of mitral annular displacement and velocity in patients with various heart conditions. Eur Heart J Cardiovasc Imaging. 2014;15(1):62-69.

14. Sengupta PP, Tajik AJ, Chandrasekaran K, Khandheria BK. Twist mechanics of the left ventricle: principles and application. JACC Cardiovasc Imaging. 2008;1(3):366376.

15. Russel IK, Gotte MJ, Bronzwaer JG, Knaapen P, Paulus WJ, van Rossum AC. Left ventricular torsion: an expanding role in the analysis of myocardial dysfunction. JACC Cardiovasc Imaging. 2009;2(5):648-655.

16. Nagueh SF, Smiseth OA, Appleton CP, Byrd BF, 3rd, Dokainish H, Edvardsen T, Flachskampf FA, et al. Recommendations for the evaluation of left ventricular diastolic function by echocardiography: an update from the American society of echocardiography and the European association of cardiovascular imaging. J Am Soc Echocardiogr. 2016;29(4):277-314. 